\title{
Parameter Identification and Synchronization of Uncertain Chaotic Systems Based on Sliding Mode Observer
}

\author{
Li-lian Huang and Lei Lin \\ Harbin Engineering University, Harbin, Heilongjiang 150001, China \\ Correspondence should be addressed to Li-lian Huang; lilian_huang@163.com
}

Received 25 July 2013; Accepted 6 September 2013

Academic Editor: Guanghui Sun

Copyright ( 2013 L.-1. Huang and L. Lin. This is an open access article distributed under the Creative Commons Attribution License, which permits unrestricted use, distribution, and reproduction in any medium, provided the original work is properly cited.

The synchronization of nonlinear uncertain chaotic systems is investigated. We propose a sliding mode state observer scheme which combines the sliding mode control with observer theory and apply it into the uncertain chaotic system with unknown parameters and bounded interference. Based on Lyapunov stability theory, the constraints of synchronization and proof are given. This method not only can realize the synchronization of chaotic systems, but also identify the unknown parameters and obtain the correct parameter estimation. Otherwise, the synchronization of chaotic systems with unknown parameters and bounded external disturbances is robust by the design of the sliding surface. Finally, numerical simulations on Liu chaotic system with unknown parameters and disturbances are carried out. Simulation results show that this synchronization and parameter identification has been totally achieved and the effectiveness is verified very well.

\section{Introduction}

Chaos has the class-random characteristics, so it has the great application prospects in the secure communications. There has been significant interest in using chaotic dynamics to realize secure communications and cryptography during the last two decades. Since Carroll and Pecora [1] and Liv et al. [2] first put forward and achieved the synchronization of drive-response chaotic systems, more and more people have aroused a great enthusiasm in the chaotic synchronization research [3-9]. In recent years people have proposed many synchronization methods of chaotic systems such as linear and nonlinear feedback control [10], delayed feedback control [11], adaptive synchronization control [12], neural network control [13], pulse control [14], and sliding mode variable structure control [15-17]. Xiu-chun et al. [18] do the research based on drive system with unknown parameters or external disturbances but do not take into account the robustness issues of the system. Therefore, regarding how to control effectively and achieve synchronization of chaotic systems with unknown parameters or interference has more practical significance.
Most of the work in this area focused on synchronization of chaotic systems to recover information signals [19-21]. Gao et al. [22] proposed a method to achieve the generalized synchronization based on state observer and pole placement. Liu et al. [23] achieved synchronization for a class of chaotic systems by nonlinear observer. Hua et al. [24] designed adaptive observer to achieve system synchronization by assuming the system parameters are given. However, not all the states of nonlinear systems can be realized. In this case, state observer can be used for observing the state to obtain the actual values and apply them to control the system. So a full-state feedback observation has been applied widely in modern control. Based on the state observer, synchronization of chaotic systems can be achieved too.

Sliding mode variable structure control [25-28] has a history of more than 50 years and it is a special nonlinear control. Its structure is not fixed and can change with a dynamic process according to the current state of the system. It is widely applied in linear and nonlinear system, continuous and discrete system, certainty and uncertainty system, and so forth and gradually extended to practical engineering applications. 
Since the sliding mode can be designed and has robustness for parameters and disturbances, which makes variable structure have fast response, be insensitive to parameter variations and disturbances, not need online identification for a system and have simple physical implementation. So the sliding mode variable structure control attracts the attention widely.

In this paper, based on sliding mode variable structure control theory and observer, a sliding mode variable structure observer is obtained to achieve the synchronization of uncertain chaotic systems with unknown parameters and external disturbances. According to the Lyapunov stability theory, the condition of synchronization and proof is presented. We point out the capability of the sliding mode observer to handle disturbances, as these have shown to be a challenge for other observers. It also has stronger robustness for chaotic systems and could accurately identify the unknown parameters with external disturbances. The observer has fewer constraints and wide application, which is suitable for most common chaotic systems. Finally, numerical simulation by Liu chaotic system verifies the effectiveness of this method.

\section{System Model and Observer Design}

2.1. Chaotic System Model. Consider the following nonlinear chaotic system model:

$$
\begin{gathered}
\dot{x}(t)=A x(t)+f(x, t)+\delta(x, t) \theta+B d(t), \\
y(t)=C x(t),
\end{gathered}
$$

where $x(t) \in R^{n}, y(t) \in R^{p}$ are the state variable and output of the system, $A \in R^{n \times n}, B \in R^{n \times m}$, and $C \in R^{p \times n}$ $(n>p>m)$ are known constant matrixes, and $(A, C)$ is observable. $f(x, t): R^{n} \rightarrow R^{n}, \delta(x, t): R^{n} \rightarrow R^{p \times q}$ are the nonlinear parts; they satisfy the conditions of Lipschitz. $\theta \in R^{n}$ is the unknown parameter vector and $\|\theta\| \leq \varepsilon_{\theta}, d(t)$ is the disturbance and $\|d(t)\| \leq \rho$, where $\rho$ is a positive constant. The expression (1) represents most common model of the chaotic systems. When $\delta(x, t)=0$, formula (1) represents a class of chaotic systems with known parameters. Liu et al. chaotic system [29], Chen chaotic system, and Lorenz chaotic system can be the form of the above model.

We make the following assumptions.

(A1) $(A, C)$ is observable so there is an observer gain $K \in$ $R^{n \times p}$ such that $A_{0}=A-K C$ is strictly Hurwitz matrix.

(A2) The known nonlinearity $f(x, t), \delta(x, t)$ satisfies

$$
\begin{aligned}
& \|f(x, t)-f(\widehat{x}, t)\| \leq \gamma_{1}\|x-\widehat{x}\| \\
& \|\delta(x, t)-\delta(\widehat{x}, t)\| \leq \gamma_{2}\|x-\widehat{x}\|,
\end{aligned}
$$

where $\gamma_{1}, \gamma_{2} \in R^{+}$are Lipschitz constants.

(A3) There is $F \in R^{m \times p}$; it makes $P B=C^{T} F^{T}$ hold.

(A4) There are positive definite matrixes $P \in R^{n \times n}, Q \in$ $R^{n \times n}$ which satisfy the Lyapunov equation $A_{0}^{T} P+$ $P A_{0}=-Q$.
2.2. Observer Design. Based on the previous assumptions, for the drive system (1) the design of sliding mode observer is as follows:

$$
\begin{gathered}
\dot{\hat{x}}(t)=A \widehat{x}(t)+f(\widehat{x}, t)+\delta(\widehat{x}, t) \hat{\theta}+K(y-C \widehat{x})+v, \\
\widehat{y}(t)=C \widehat{x}(t) .
\end{gathered}
$$

$\widehat{x}(t) \in R^{n}$ is the state variable of the observer. $\widehat{y}(t)$ is output of the observer. $\hat{\theta} \in R^{n}$ is the estimation of parameter $\theta$. $K$ is the observer gain matrix. $v$ is the nonlinear input.

The state error vector is $e=x-\widehat{x}$ between drive system (1) and response system (3), so the error system equation can be obtained as follows:

$$
\begin{aligned}
\dot{e}=( & A-K C) e+f(x, t)-f(\widehat{x}, t)+\delta(x, t) \theta \\
& -\delta(\widehat{x}, t) \hat{\theta}+B d(t)-v .
\end{aligned}
$$

The goal of the design is for a class of chaotic systems (1) with unknown parameters and external disturbance, we search the right gain matrix $K$ and nonlinear input $v$ and design the observer by sliding mode variable structure control theory to achieve the synchronization of chaotic systems.

If the error states in system (4) converge to the origin asymptotically or in a limited time, the synchronization of the response system (3) and drive system (1) is achieved.

In order to design the sliding mode variable structure observer (3), the sliding mode surface is designed as the form $s=F C e=F(y-C \widehat{x})$ and nonlinear input $v$ is defined as (5) in discrete item.

$$
v= \begin{cases}\frac{P^{-1} C^{T} F^{T} F C e}{\|F C e\|} \beta, & \|F C e\| \neq 0, \beta>\rho \\ 0, & \|F C e\|=0,\end{cases}
$$

Theorem 1. Considering the system (1) and (3), under the condition of satisfying the assumptions 1-4, nonlinear input (5) is chosen and it satisfies

$$
\gamma_{1}+\varepsilon_{\theta} \gamma_{2} \leq \frac{1}{2} \frac{\lambda_{\min }(Q)}{\lambda_{\max }(P)}
$$

the parameter update rules are chosen as

$$
\dot{\hat{\theta}}=n \delta^{T}(\widehat{x}, t) P(x-\widehat{x}) \text {. }
$$

There must be $\lim _{t \rightarrow \infty}\|e\|=0$ and $\lim _{t \rightarrow \infty} \widehat{\theta}=\theta$ for any initial conditions, namely, the system (1) and (3) realized the chaos synchronization and parameter estimation.

Proof. Define the parameter error vector $\widetilde{\theta}=\widehat{\theta}-\theta$, and select Lyapunov function as follows,

$$
V=e^{T} P e+\frac{1}{n} \widetilde{\theta}^{T} \widetilde{\theta}
$$


Then its derivative is

$$
\begin{aligned}
\dot{V}= & e^{T} P e+e^{T} P \dot{e}+\frac{1}{n} \dot{\tilde{\theta}}^{T} \tilde{\theta}+\frac{1}{n} \widetilde{\theta}^{T} \dot{\tilde{\theta}} \\
= & e^{T}\left(A_{0}^{T} P+P A_{0}\right) e+2 e^{T} P(f(x, t)-f(\widehat{x}, t)) \\
& +2 e^{T} P \delta(x, t) \theta \\
& -2 e^{T} P \delta(\widehat{x}, t) \hat{\theta}+2 e^{T} P B d(t)-2 e^{T} P v+\frac{2}{n} \widetilde{\theta}^{T} \dot{\tilde{\theta}} \\
= & -e^{T} Q e+2 e^{T} P(f(x, t)-f(\widehat{x}, t)) \\
& +2 e^{T} P(\delta(x, t)-\delta(\widehat{x}, t)) \theta \\
& +2 e^{T} P B d(t)-2 e^{T} P v+\frac{2}{n} \widetilde{\theta}^{T} \dot{\tilde{\theta}}-2 e^{T} P \delta(\widehat{x}, t) \widetilde{\theta} \\
= & -e^{T} Q e+2 e^{T} P(f(x, t)-f(\widehat{x}, t)) \\
& +2 e^{T} P(\delta(x, t)-\delta(\widehat{x}, t)) \theta \\
& +2 e^{T} P B d(t)-2 e^{T} P v \\
\leq & -\lambda_{\min }(Q)\|e\|^{2}+2 \gamma_{1}\|e\| \cdot\|P\| \cdot\|e\| \\
& +2 \gamma_{2}\|e\| \cdot\|P\| \cdot\|\theta\| \cdot\|e\|+2 e^{T} P B\|d(t)\|-2 e^{T} P v \\
\leq & -\lambda_{\min }(Q)\|e\|^{2}+2 \gamma_{1} \lambda_{\max }(P)\|e\|^{2}+2 \varepsilon_{\theta} \gamma_{2} \lambda_{\max }(P)\|e\|^{2} \\
& +2\|F C e\| \rho-2 e^{T} P \frac{P^{-1} C^{T} F^{T} F C e}{\|F C e\|} \beta \\
= & -\left(\lambda_{\min }(Q)-2 \gamma_{1} \lambda_{\max }(P)-2 \varepsilon_{\theta} \gamma_{2} \lambda_{\max }(P)\right)\|e\|^{2} \\
& +2\|F C e\|(\rho-\beta) .
\end{aligned}
$$

According to Theorem $1 \gamma_{1}+\varepsilon_{\theta} \gamma_{2} \leq(1 / 2) \lambda_{\min }(Q) / \lambda_{\max }(P)$ and the definition of the nonlinear input $v$, we get

$$
\begin{aligned}
\dot{V} & \leq-\left(\lambda_{\min }(Q)-2 \gamma_{1} \lambda_{\max }(P)-2 \varepsilon_{\theta} \gamma_{2} \lambda_{\max }(P)\right)\|e\|^{2} \\
& =-\xi\|e\|^{2} \leq 0,
\end{aligned}
$$

where $\xi=\lambda_{\min }(Q)-2 \gamma_{1} \lambda_{\max }(P)-2 \varepsilon_{\theta} \gamma_{2} \lambda_{\max }(P)>0$ and $\lambda_{\max }(\cdot), \lambda_{\min }(\cdot)$ are the maximum and minimum eigenvalues of the matrix $(\cdot)$.

According to Lyapunov stability theory, the original Lyapunov function is positive $V(x) \geq 0$ and its first derivative is negative $\dot{V}(x) \leq 0$, then the error system $e(t)$ converges to zero. So the expression (1) and (3) achieve synchronization finally.

Remark 2. In the design of the observer, it is very important that the assumption 4 is satisfied or not. When the matrix $P \in R^{n \times n}$ satisfies the assumption 4 , the sufficient condition is: The transfer function $G_{F}(s)=F C(s I-(A-K C))^{-1} B$ which contains gain matrix $K$ is strictly positive.
Remark 3. Discontinuous switch $v$ can make the observer error produce a sliding motion and also the observer has the robustness for the unknown input disturbances. However this discontinuous switch will cause some shocks. In order to eliminate this shake which is caused by high frequency interference, $v$ can be substituted by $v=\left(P^{-1} C^{T} F^{T} F C e\right) /(\|F C e\|+$ $\mu) \beta$, where $\mu$ is a small scalar quantity.

\section{Simulation Example}

In order to verify the correctness of the above method, Liu chaotic system is an example to simulate. In this paper, we study the Liu which is a typical chaotic system with characteristics of chaos and its expression is

$$
\begin{aligned}
& \dot{x}_{1}=a\left(x_{2}-x_{1}\right), \\
& \dot{x}_{2}=b x_{1}-d x_{1} x_{3}, \\
& \dot{x}_{3}=m x_{1}^{2}-c x_{3} .
\end{aligned}
$$

$a, b, c, d, m$ are the system parameters. When $a=10, b=$ $40, c=2.5, d=10$, and $m=4$, the Lyapunov exponents of Liu system are $\lambda_{1}=1.6393$, and $\lambda_{2}=0, \lambda_{3}=-14.1305$. Therefore, under certain initial conditions Liu system is in a chaotic state and presents a rich and complex behavior of chaotic dynamics.

Assume that the parameters $a, b, c$ of Liu chaotic system are unknown, so the Liu chaotic system with unknown parameters and disturbance is described as follows:

$$
\begin{gathered}
\dot{x}(t)=A x(t)+f(x, t)+\delta(x, t) \theta+B d(t), \\
y(t)=C x(t),
\end{gathered}
$$

where $x=\left[\begin{array}{lll}x_{1} & x_{2} & x_{3}\end{array}\right]^{T}$ is the state variable, $y$ is the output, $f(x, t)=\left[\begin{array}{c}-x_{2} \\ -x_{3}-d x_{1} x_{3} \\ m x_{1}^{2}-x_{1}\end{array}\right], \delta(x, t)=\left[\begin{array}{ccc}x_{2}-x_{1} & 0 & 0 \\ 0 & x_{1} & 0 \\ 0 & 0 & -x_{3}\end{array}\right]$, parameter vector is $\theta=\left[\begin{array}{l}a \\ b \\ c\end{array}\right], A=\left[\begin{array}{lll}0 & 1 & 0 \\ 0 & 0 & 1 \\ 1 & 0 & 0\end{array}\right], C=\left[\begin{array}{l}1 \\ 0 \\ 0\end{array}\right]^{T}$. It is easy to verify that $(A, C)$ can be observable.

Equation (12) is the drive system and the observer is designed:

$$
\begin{gathered}
\dot{\hat{x}}(t)=A \hat{x}(t)+f(\widehat{x}, t)+\delta(\widehat{x}, t) \hat{\theta}+K(y-C \widehat{x})+v, \\
\hat{y}(t)=C \widehat{x}(t) .
\end{gathered}
$$

In simulation, the initial value of Liu drive system and its observer are chosen as $x(0)=\left[\begin{array}{lll}1 & 3 & 5\end{array}\right]^{T}$, and $\widehat{x}(0)=$ $\left[\begin{array}{lll}2 & 3 & 7\end{array}\right]^{T}$. The initial value of the unknown parameters are chosen as $\hat{\theta}(0)=\left[\begin{array}{lll}2.1 & 1.1 & 0.1\end{array}\right]^{T}$. The disturbance is $d(t)=$ $0.3 \sin (2 t),\|d(t)\| \leq \rho=0.3$. The constant parameters are chosen as $\beta=0.5, \gamma_{1}=0.01, \gamma_{2}=0.0002$, and $\varepsilon_{\theta}=45$. Select the observer gain matrix $K=\left[\begin{array}{lll}2 & 1 & 2\end{array}\right]^{T}$, so that the eigenvalues of $A-K C$ are $-1.7549,-0.1226+0.7449 i$, and $-0.1226-0.7449 i$, respectively. The parameter vector updates as $\dot{\hat{\theta}}=n\left[\begin{array}{ccc}x_{2}-x_{1} & 0 & 0 \\ 0 & x_{1} & 0 \\ 0 & 0 & -x_{3}\end{array}\right] P(x-\hat{x})$. Choose positive 


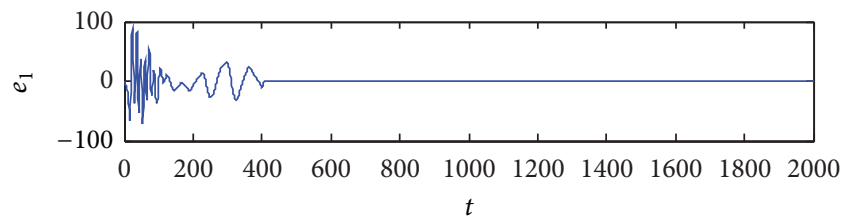

(a)

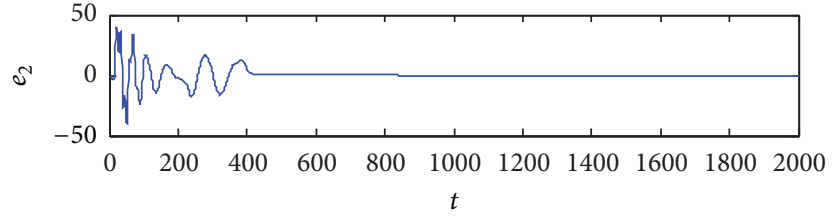

(b)

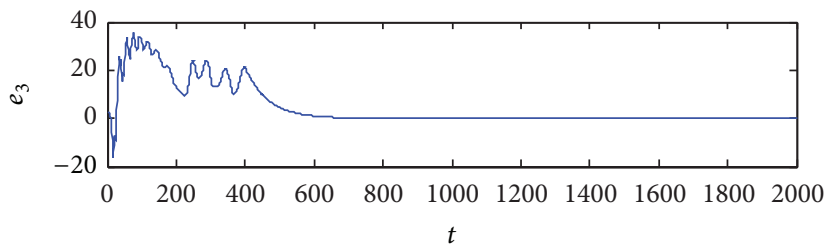

(c)

FIGURE 1: Graph of error state $e_{1}, e_{2}, e_{3}$.

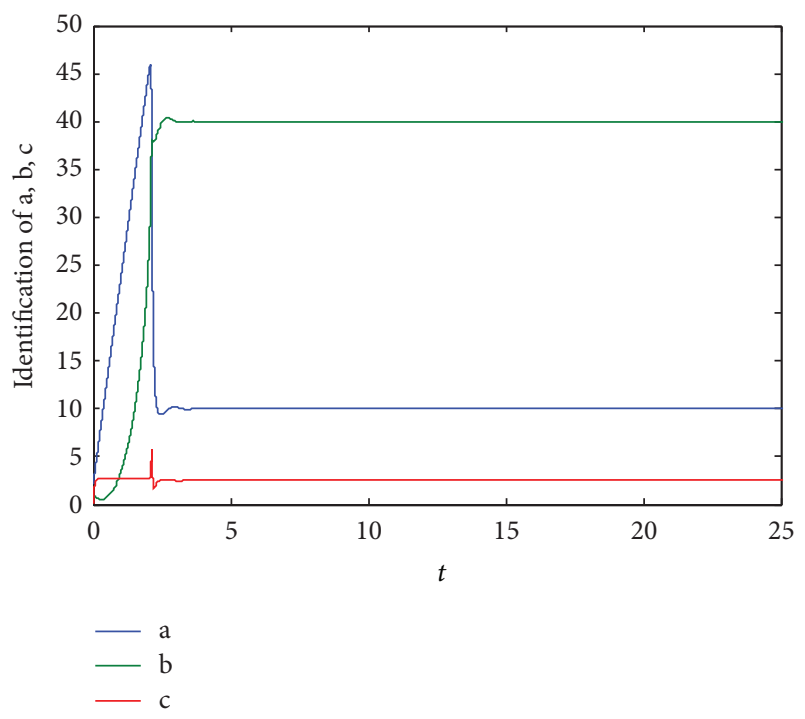

FIGURE 2: Graph of parameter identification.

definite symmetric matrix $P=\left[\begin{array}{ccc}3 & -1 & -2 \\ -1 & 3 & -1 \\ -2 & -1 & 5\end{array}\right], Q=\left[\begin{array}{ccc}6 & -3 & 1 \\ -3 & 2 & -1 \\ 1 & -1 & 2\end{array}\right]$, $B=[(2 / 3)(1 / 3)(1 / 3)]^{T}$, and $F=1$. The time step is $h=0.001$. The above parameters and the matrixes are applied to the observer of the Liu system. We can obtain Figures 1 and 2. Figure 1 is the graph of the error state. From the figure we can see that by adding the observer when $N=400$ the error system quickly converges to zero and achieves the synchronization. Figure 2 is the graph of the parameter identification. From the figure we can obtain that the parameters of system approaches the true value. The simulation results verify the effectiveness of the method.

\section{Conclusions}

The state observer based on sliding mode control is proposed to synchronize a class of chaotic systems with uncertain parameters and external disturbance and identification method for unknown parameter is given. Nonlinear parts satisfy the Lipschitz condition and external disturbance is bounded in this uncertain nonlinear system which is discussed. Depending on characteristics of the system, four assumptions are given. In the condition of satisfying the assumptions, a sliding mode observer is designed by combining sliding mode variable structure control and observer methods, and the synchronization of chaotic systems is realized finally. By means of Lyapunov stability theory, the conditions to realize the synchronization of the disturbed chaotic system with uncertain parameters is given and the correctness of the observer is verified. The numerical simulations by Liu chaotic system show that the designed sliding mode observer can still effectively realize the observation of the state variables and identification of the unknown parameters. Simulation results verify the effectiveness of the proposed methods. The observer has stronger robustness to chaotic systems with unknown parameters or external disturbance and has fewer constraints and wide application, which is suitable for most common chaotic systems.

\section{Acknowledgments}

This research is supported by the Chinese National Natural Science Foundation (no. 60772025) and the National Natural Science Foundation of Heilongjiang province (Grant no. F201220).

\section{References}

[1] T. L. Carroll and L. M. Pecora, "Synchronizing chaotic circuits," IEEE Transactions on Circuits and Systems I, vol. 38, pp. 453456, 1991.

[2] F. Liu, Y. Ren, X. Shan, and Z. Qiu, "A linear feedback synchronization theorem for a class of chaotic systems," Chaos, Solitons \& Fractals, vol. 13, no. 4, pp. 723-730, 2002.

[3] H. Li-Lian and H. Shao-Jie, "Stability of fractional state space system and its application to fractional order chaotic system," Acta Physica Sinica, vol. 60, no. 4, Article ID 044703, 2011. 
[4] Y. Tang and W. K. Wong, "Distributed synchronization of coupled neural networks via randomly occurring control," IEEE Transactions on Neural Networks and Learning Systems, vol. 24, no. 3, pp. 435-447, 2013.

[5] L. L. Huang and N. Ma, "A new method for projective synchronization of different fractional order chaotic systems," Acta Physica Sinica, vol. 61, no. 16, Article ID 160510, 2012.

[6] Y. Tang, Z. Wang, H. Gao, S. Swift, and J. Kurths, "A constrained evolutionary computation method for detecting controlling regions of cortical networks," ACM/IEEE Transactions on Computational Biology and Bioinformatics, vol. 9, no. 6, pp. 15691581, 2012.

[7] B. R. Andrievskiǔ, V. O. Nikiforov, and A. L. Fradkov, "Adaptive observer-based synchronization of the nonlinear nonpassifiable systems," Automation and Remote Control, vol. 68, no. 7, pp. 1186-1200, 2007.

[8] Y. Tang, H. Gao, W. Zou, and J. Kurths, "Distributed synchronization in networks of agent systems with nonlinearities and random switchings," IEEE Transactions on Cybernetics, vol. 43, no. 1, pp. 358-370, 2013.

[9] H. Li-Lian and Q. Xue, "The synchronization of fractional order chaotic systems with different orders based on adaptive sliding mode control," Acta Physica Sinica, vol. 62, no. 8, Article ID 080507, 2013.

[10] A. Naceri N M Mansouri and A. Charef, "Prediction-based feedback control of fractional order system," in Proceedings of the IEEE International Symposium on Industrial Electronics, (ISIE '08), pp. 908-912, 2008.

[11] K.. E. Chlouverakis and J. C. Sprott, "Chaotic hyperjerk systems," Chaos Solitons and Fractals, no. 28, pp. 739-746, 2006.

[12] R. Marino and P. Tomei, "Global adaptive output-feedback control of nonlinear systems. I. Linear parameterization," IEEE Transactions on Automatic Control, vol. 38, no. 1, pp. 17-32, 1993.

[13] J. Lü and G. Chen, "A time-varying complex dynamical network model and its controlled synchronization criteria," IEEE Transactions on Automatic Control, vol. 50, no. 6, pp. 841-846, 2005.

[14] A. Kozlov, G. Osipov, and V. Shalfeer, "Suprpesing Chaos in continuos systems by impluse control," in Proceedings of the 1st Intematinal Conference on Control of Oscillations and Chaos, vol. 3, pp. 578-5581, 1997.

[15] L. Wu, C. Wang, and Q. Zeng, "Observer-based sliding mode control for a class of uncertain nonlinear neutral delay systems," Journal of the Franklin Institute, vol. 345, no. 3, pp. 233-253, 2008.

[16] X. Hu, L. Wu, C. Hu, and H. Gao, "Adaptive sliding mode tracking control for a flexible air-breathing hypersonic vehicle," Journal of the Franklin Institute, vol. 349, no. 2, pp. 559-577, 2012.

[17] R. Raoufi and A. S. I. Zinober, "Smooth adaptive sliding mode observers in uncertain chaotic communication," International Journal of Systems Science, vol. 38, no. 11, pp. 931-942, 2007.

[18] L. Xiu-Chun, G. Jian-Hua, W. Yun-Lan, and Z. Tian-Hai, "Observer synchronization method for a class of perturbed chaotic systems with unknown parameters," Acta Physica Sinica, vol. 60, no. 3, Article ID 030505, 2011.

[19] Y. Wu, X. B. Zhou, J. Chen, and B. Hui, "Chaos synchronization of a new 3D chaotic system," Chaos Solitons and Fractals, vol. 42, pp. 1812-1819, 2009.

[20] M. Hasler and Y. L. Maistrenko, "An introduction to the synchronization of chaotic systems: coupled skew tent maps," IEEE Transactions on Circuits and Systems I, vol. 44, no. 10, pp. 856-866, 1997.
[21] L. Kocarev, K. S. Halle, K. Eckert, and L. O. Chua, "Experimental demonstration of secure communication via chaotic synchronization," International Journal of Bifurcation and Chaos, vol. 2, pp. 709-713, 1992.

[22] G. GaoT, Z. Q. Chen, Z. Z. Yuan, and Q. L. Gu, "Study on synchronization of chaotic systems based on observer," Acta Physica Sinica, vol. 53, no. 5, pp. 1305-1308, 2004.

[23] F. Liu, Y. Ren, X. M. Shan, and Z. L. Qiu, "Synchronization for a class of chaotic systems based upon observer theory," Chinese Physics, vol. 10, article 606, 2001.

[24] C. Hua, X. Guan, X. Li, and P. Shi, "Adaptive observer-based control for a class of chaotic systems," Chaos, Solitons \& Fractals, vol. 22, no. 1, pp. 103-110, 2004.

[25] L. Wu and W. X. Zheng, "Passivity-based sliding mode control of uncertain singular time-delay systems," Automatica, vol. 45, no. 9, pp. 2120-2127, 2009.

[26] L. Wu, P. Shi, and H. Gao, "State estimation and slidingmode control of Markovian jump singular systems," IEEE Transactions on Automatic Control, vol. 55, no. 5, pp. 1213-1219, 2010.

[27] L. Wu, D. W. C. Ho, and C. W. Li, "Sliding mode control of switched hybrid systems with stochastic perturbation," Systems \& Control Letters, vol. 60, no. 8, pp. 531-539, 2011.

[28] L. Wu and H. Gao, "Sliding mode control of two-dimensional systems in Roesser model," IET Control Theory \& Applications, vol. 2, no. 4, pp. 352-364, 2008.

[29] C. Liu, T. Liu, L. Liu, and K. Liu, "A new chaotic attractor," Chaos, Solitons and Fractals, vol. 22, no. 5, pp. 1031-1038, 2004. 


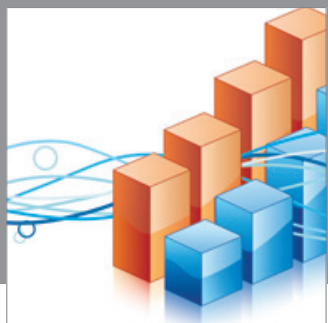

Advances in

Operations Research

mansans

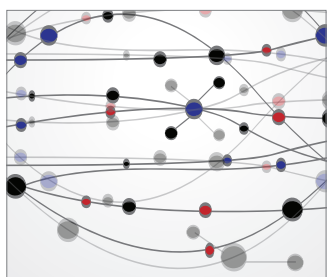

The Scientific World Journal
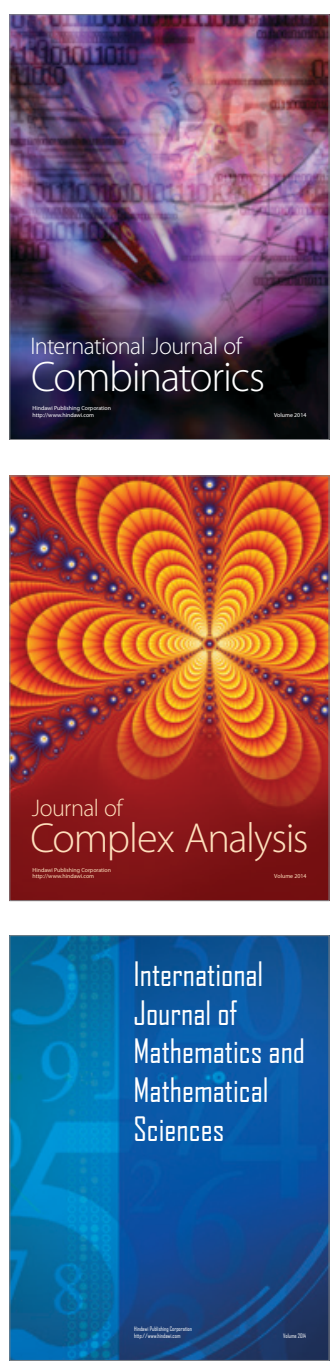
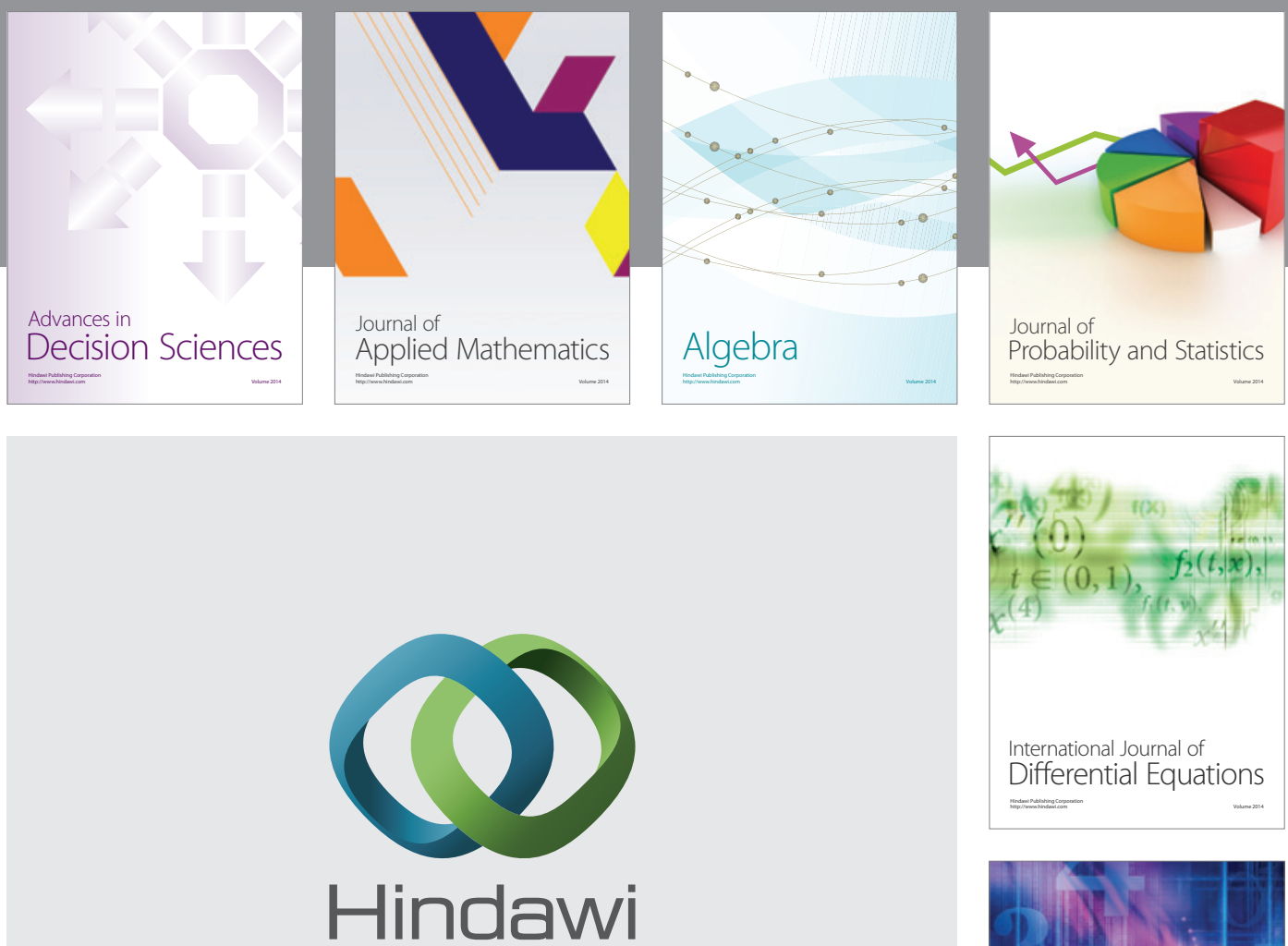

Submit your manuscripts at http://www.hindawi.com
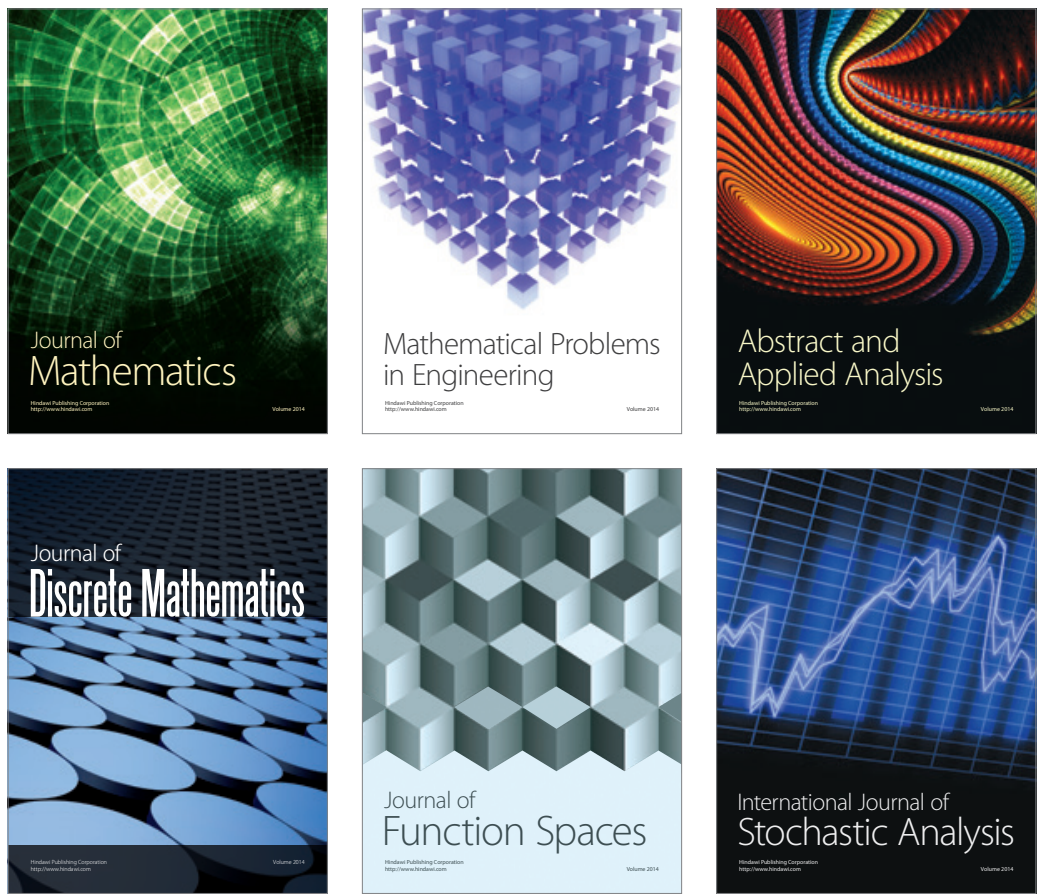

Journal of

Function Spaces

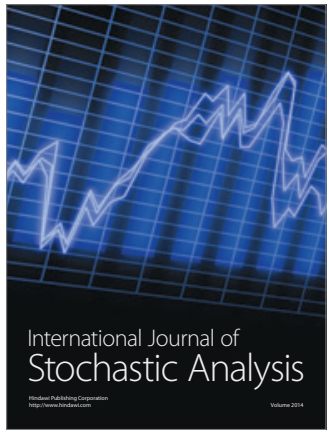

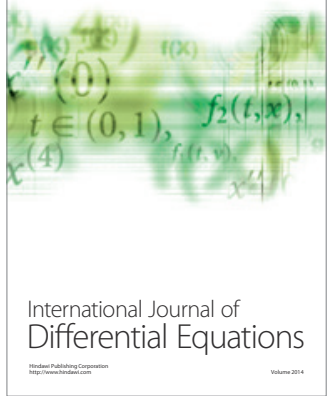
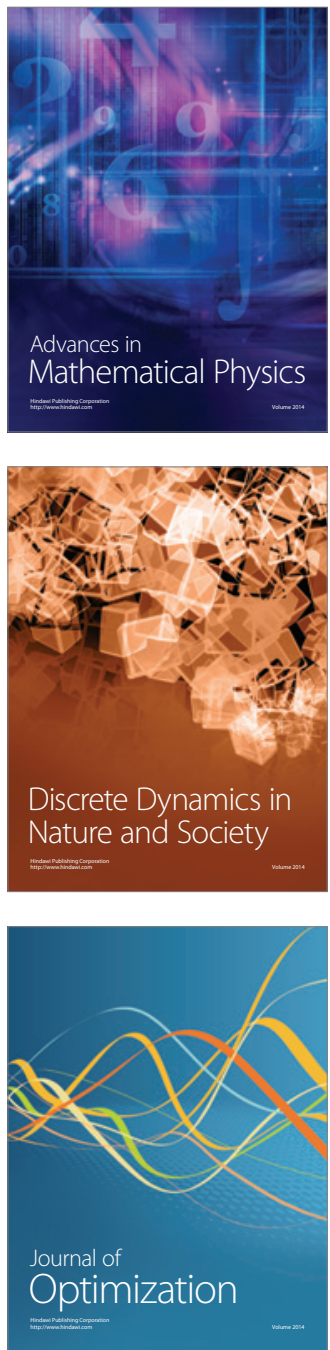